\title{
Antibody Conjugated Oligonucleotides as a Platform for Cyclic Immunofluorescent Staining
}

Nathan McMahon ${ }^{1}$, Jocelyn Jones ${ }^{1}$, Allison Solanki ${ }^{1}$, Jennifer Eng ${ }^{1}$, Sunjong Kwon ${ }^{1}$, Young-Hwan Chang $^{1,4}$, Guillaume Thibault ${ }^{1,4}$, Koei Chin ${ }^{1,3}$, Michel Nederlof ${ }^{5}$, Joe Gray ${ }^{1,2,3}$ and Summer L. Gibbs ${ }^{1,2,3}$

${ }^{1 .}$ Department of Biomedical Engineering, Oregon Health and Science University, Portland, OR, USA.

2. Knight Cancer Institute, Oregon Health and Science University, Portland, OR, USA.

3. Center for Spatial Systems Biomedicine, Oregon Health and Science University, Portland, OR, USA.

4. Computational Biology Program, Oregon Health and Science University, Portland, OR, USA.

5. Quantitative Imaging Systems, Pittsburg, PA, USA.

* Corresponding author: mcmahonn@ohsu.edu

Significant tumor heterogeneity is driven by complex interactions between cell populations, as well as cross talk between signaling pathways, has largely foiled modern medicine's efforts towards successful cancer cures. Advances in molecular characterization technologies, such as next generation sequencing, have vastly affected how cancer is diagnosed and treated such as identifying subtypes within breast cancer $[1,2]$. Genomic analyses are a powerful therapeutic tool, but at the cost of cellular and subcellular context of biomarker distribution. Recent discoveries, have revealed the significance of the spatial relationships of cancer, immune, and microenvironmental cells $[3,4]$. To further understand the diagnostic and prognostic implications of these relationships, a molecular profiling technology to measure both expression and spatial context of biomarkers, while preserving both will be required.

Histopathology, most commonly performed on formalin-fixed paraffin-embedded (FFPE) tissue, is one of the most important tools for pathological diagnosis. Conventional methods of immunohistochemical (IHC) and immunofluorescent (IF) staining are typically limited to labeling 2-5 antigens on a single sample [5], making translation of genomic analyses to conventional pathological diagnosis impossible. There have been a number of strategies proposed to increase specific antigen labeling on a single sample, all of which are unable to be readily integrated into routine clinical histopathology [5-9]. This limitation has led to the development of techniques using serial cycles of fluorescent tagging, imaging, and bleaching [9] or dissociation of affinity tags [5,7] to vastly increase imaging dimensionality. These technologies greatly improve the multiplexing capabilities of IF, but will be difficult to integrate into clinical histopathology as tissue antigenicity and integrity are affected by destaining methods [10].

Our improved, highly-multiplexable, cyclic IF (CycIF) technique is capable of generating extreme multiparametric images for quantifying biomarker expression and distribution. Termed antibody conjugated oligonucleotide (Ab-oligo) CycIF, it preserves tissue antigenicity and lends itself to ready integration into clinical workflows. Ab-oligo CycIF exploits in situ hybridization of complementary oligonucleotides for biomarker labeling and the oligo modifications to facilitate signal removal for sequential rounds of fluorescent tagging. In our technology, a single stranded oligo (docking strand, DS) is conjugated to the primary antibody. Then the introduction of a complementary single stranded oligo (imaging strand, IS) conjugated to a conventional fluorophore, facilitates specific on tissue fluorescence labeling through in situ hybridization which can be imaged using any conventional fluorescence microscope. Signal removal can be completed using a variety of strategies, which result in full signal removal to levels of autofluorescence prior to subsequent staining cycles (Fig. 1A). Using Ab-oligo CycIF, we have generated up to 12 color images on multiple HER2 positive breast cancers to validate our technology. 
Combining Ab-oligo CycIF with our novel intracellular paired agent imaging (iPAI) technology will enable quantification of drug distribution and therapeutic responses using molecularly targeted therapies. iPAI uses spectrally distinct, fluorescently labeled targeted and untargeted drugs, correcting for untargeted uptake and facilitating quantitative assessment of the treated phosphoproteome [11,12]. iPAI can be used to accurately calculate drug target availability (DTA), drug target occupancy (DTO) or engagement and drug phosphorylation response (DPR) (Fig. 2B). Following iPAI imaging, Ab-oligo CycIF can be applied to interrogate changes to heterogenous cell populations within tumors that drive variable therapeutic responses in patients and develop personalized medicine.

\section{References:}

[1] Nature 490(7418) (2012), p. 61.

[2] T Sørlie et al., Proc. Natl. Acad. Sci. U. S. A. 98(19) (2001), p. 10869.

[3] A Mezheyeuski et al., J. Pathol. 244(4) (2018), p. 421.

[4] WH Fridman et al., Nat. Rev. Cancer 12(4) (2012), p. 298.

[5] EC Stack et al., Methods 70(1) (2014), p. 46.

[6] H Tsurui et al., J. Histochem. Cytochem. 48(5) (2000), p. 653.

[7] P Zrazhevskiy, LD True and X Gao, Nat. Protoc. 8(10) (2013), p. 1852.

[8] T Tsujikawa et al., Cell Rep. 19(1) (2017), p. 203.

[9] J-R Lin, M Fallahi-Sichani and PK Sorger, Nat. Commun. 6 (2015), p. 8390.

[10] "SIMPLE: A Sequential Immunoperoxidase Labeling and Erasing Method.", https://www.ncbi.nlm.nih.gov/pmc/articles/PMC2746723/(17 January 2019).

[11] KS Samkoe et al., Cancer research 74 (2014), p. 7465.

[12] KM Tichauer et al., Nature Medicine 20 (2014), p. 1348.

\section{A.}

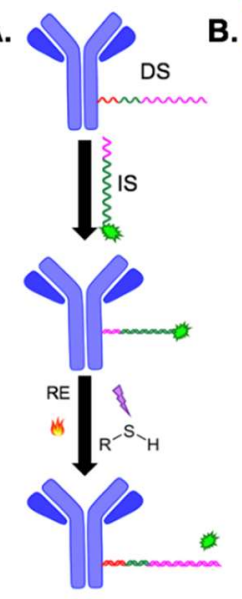

Figure 1. Ab-oligo cycIF and iPAI scheme. A. Primary antibodies conjugated to single stranded oligos (DS $=$ docking strand). After Ab-oligo tissue labeling, the complementary oligo conjugated to a fluorophore (IS = imaging strand) was used for in situ hybridization. After imaging, signal removal was completed using thermal melting, UV light exposure, RE-based oligo cleavage or use of thiol for disulfide bond cleavage. B. iPAI can quantify (1) DTA by measuring untreated samples, (2) DTO by measuring treated samples and subtracting the value from untreated samples (DTA) and (3) DPR by measuring downstream proteins on samples treated with parent drugs (TKI). 